\title{
Unexpected unrelated umbilical cord blood stem cell engraft in two patients with severe aplastic anemia that received immunosuppressive treatment: A case report and literature review
}

\author{
LIN-NA XIE and FANG ZHOU
}

Department of Hematology, The General Hospital of Jinan Military, Jinan, Shandong 250031, P.R. China

Received September 3, 2014; Accepted July 17, 2015

DOI: $10.3892 / \mathrm{etm} .2015 .2698$

\begin{abstract}
Severe aplastic anemia (SAA) is a life-threatening bone marrow disorder. Bone marrow transplantation is the primary therapy for SAA; however, its efficacy is limited by numerous factors, including lack of histocompatible sibling donor, patient age and graft-versus-host-disease (GVHD) following transplantation. Immunosuppressive treatment (IST) is the first procedure developed for patients without a sibling donor. Our previous study reported that patients administered enhanced IST, in addition to a regime of unrelated umbilical cord blood (UCB) transfusion, exhibited higher efficiency and a reduced rate of relapse. Therefore, the present study reported the cases of 2 patients that received enhanced IST plus unrelated UCB transfusion. These patients exhibited complete hematological recovery with an increased rate of mixed chimerism and demonstrated no signs of GVHD or relapse during the 2-year follow-up period. Thus, enhanced immunosuppressive treatment (low-dose cyclophosphamide and antithymocyte globulin) combined with UCB transfusion may be an effective treatment for patients with SAA.
\end{abstract}

\section{Introduction}

Aplastic anemia (AA) is a serious disorder characterized by pancytopenia and hypocellular bone marrow. Severe AA (SAA) is diagnosed when 2 of 3 blood parameters meet the following criteria: Absolute neutrophil count, $<500 / \mathrm{ml}$; absolute reticulocyte count, $<60,000 / \mathrm{ml}$; and platelet count, $<20,000 / \mathrm{ml}$ (1). AA is a disease that may present at any age, and SAA is always fatal if untreated. Adolescent and young adult patients $(\leq 30$ years old) with SAA may exhibit marrow failure, in contrast to older adult patients. Matched sibling hematopoietic stem cell transplantation (HSCT) is considered to be the primary treatment for patients with SAA (2). If a

Correspondence to: Dr Lin-Na Xie, Department of Hematology, The General Hospital of Jinan Military, 25 Shifan Road, Jinan, Shandong 250031, P.R. China

E-mail: xielinnadoctor@hotmail.com

Key words: immunosuppressive treatment, umbilical cord blood stem cell, severe aplastic anemia matched sibling donor is not available, it is necessary to screen unrelated histocompatible donors from a bone marrow library. Umbilical cord blood (UCB) is an alternative hematopoietic stem cell source for patients with SAA (3); however, low cell dose, higher risk of rejection and delayed immune recovery limit its application. In clinical practice, immunosuppressive treatment (IST) has been used as the primary procedure for patients without a compatible donor; however, the relapse rate reported in previous studies is high (4). In a previous study (5), the present authors reported that the patients that received enhanced IST plus one regime of unrelated UCB transfusion exhibited higher efficiency and a reduced rate of relapse. In the present report, two patients with SAA were successfully treated with enhanced immunosuppressive treatment combined with unrelated UCB. Unexpectedly, the patients exhibited mixed chimerism following therapy. Chimerism studies were conducted on multiple occasions following the treatment. By analyzing short tandem repeat regions, chimerism was identified as $5.2 \%$ on day 16 , which indicated low levels of donor T cell engraftment, and increasing to $74.6 \%$ on day 74. At 6 months after therapy, the chimerism level reached $75.2 \%$ and remained at $97.75 \%$ at 375 days after therapy.

\section{Case report}

Ethical approval and patient consent. The therapy protocols of the present study were approved by the Ethics Committee of the General Hospital of Jinan Military (Jinan, China). Written consent was obtained from the patient or patient's legal guardian. The patients were screened for Fanconi anemia and paroxysmal nocturnal hemoglobinuria.

Case 1. A 10-year-old boy was diagnosed with SAA in September 2012 at the General Hospital of Jinan Military. The patient had no matched related donor for HSCT. The treatment regimen consisted of $3 \mathrm{mg} / \mathrm{kg}$ rabbit anti-thymocyte globulin (ATG) from day -5 to day -1 , and $50 \mathrm{mg} / \mathrm{kg} /$ day cyclosphosphamide (CTX) from day -3 to day -2 . Cyclosporine A (CSA) was administered by intravenous infusion of $3 \mathrm{mg} / \mathrm{kg} /$ day (oral administration from day -1) to maintain a range of $150-250 \mathrm{ng} / \mathrm{ml}$. Unrelated UCB was transfused on day 0 , and a total of $4.2 \times 10^{7} / \mathrm{kg}$ nucleated cells and $0.9 \times 10^{5} / \mathrm{kg} \mathrm{CD} 34^{+}$cells were transfused. If the neutrophil count was $<0.5 \times 10^{9} / 1$, then granulocyte-colony stimulating factor (G-CSF; $5 \mathrm{mg} / \mathrm{kg}$ ) was administered; however, 


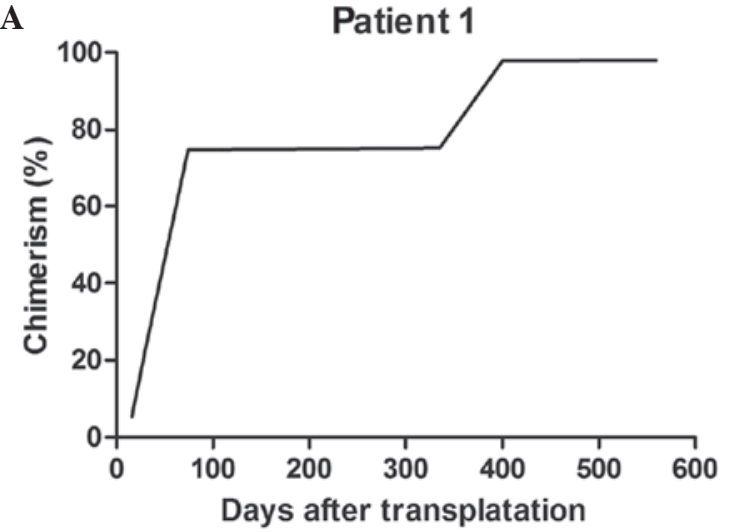

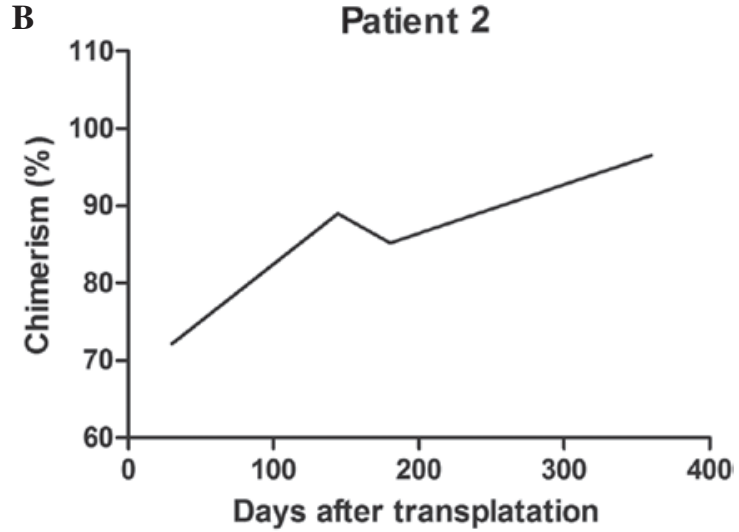

Figure 1. Chimerism of patients 1 and 2 following combined therapy.

the dose of G-CSF was gradually reduced if the neutrophil count was $>1.5 \times 10^{9} / 1$. No significant toxicity was observed. Neutrophils reached $0.5 \times 10^{9} / 1$ on day 12 and platelet counts reached $30 \times 10^{9} / 1$ on day 27 . The patient achieved complete response on day 74 and the cell phenotype of red blood cells was completely changed to the donor type at 4 months. Chimerism (through the analysis of short tandem repeat regions) was $5.2 \%$ on day 16 , increasing to $74.6 \%$ on day 74 . At 6 months after therapy, the chimerism reached $75.2 \%$ and remained at $97.75 \%$ at 375 days after therapy (Fig. 1). The latest follow-up occurred in May 2015. There was no indication of graft-versus-host disease (GVHD) or relapse, and chimerism was $100 \%$.

Case 2. A 26-year-old female was diagnosed with SAA in June 2013 and treated following a similar protocol as for case 1 . The patient was the only child in the family and had no matched related donor for HSCT. For unrelated UCB transfusion, a total of $1.7 \times 10^{7} / \mathrm{kg}$ nucleated cells and $0.36 \times 10^{5} / \mathrm{kg}$ $\mathrm{CD}_{3} 4^{+}$cells were transfused. Neutrophils reached $0.5 \times 10^{9} / 1$ on day 20 and the platelet count reached $30 \times 10^{9} / 1$ on day 32 . The patient achieved complete response on day 87 . The cell phenotype of red blood cells was completely changed to the donor type at 5 months. In addition, chimerism was $72 \%$ on day 30 , increasing to $89 \%$ on day 144 . Due to Epstein-Barr viremia, the patient discontinued the CSA therapy on day 150 , and the chimerism reduced to $85.2 \%$. Chimerism reached $100 \%$ at 361 days after the therapy (Fig. 1B). During a 2-year follow-up period, the donor chimerism remained complete implant and normal blood cell counts were detected, while there was no indication of GVHD and no relapse.

\section{Discussion}

Five decades ago, effective therapy for SAA was limited; however, advances in HSCT and IST have improved the survival rate of patients with SAA from $10-20 \%$ in the 1960 s to $80-90 \%$ at present (6). Human leukocyte antigen (HLA)-matched HSCT is recommended as the primary therapy for young patients with SAA (7); however, the lack of matched donors, graft rejection, GVHD and poor immune reconstitution limit the success of HSCT. UCB is an alternative hematopoietic stem cell source for transplantation. Hemopoietic progenitor cells (HPCs) in UCB possess extensive proliferative capacity, and the quantity of HPCs from a single UCB collection is associated with the success of bone marrow transplantation (BMT) (8). Related and unrelated UCB transplantation (UCBT) have been successfully used in the treatment of pediatric patients with various malignant or non-malignant diseases (9). Compared with BMT, UCBT is able to evidently reduce acute and chronic GVHD. However, graft rejection and poor immune reconstitution continue to limit the success rate of UCBT. A higher number of nucleated graft cells, certain conditioning regimens and the degree of mismatch between the graft and recipient are crucial for achieving improved engraftment following UCBT in patients with SAA, particularly in adult patients $(3,10)$. In order to overcome the high engraftment/alloreactivity barrier in SAA, myeloablative chemotherapy or radiation therapy have been used to induce sufficient immunosuppression or clearance of reactive recipient $\mathrm{T}$ cell populations. Total lymphoid irradiation or total body irradiation are typically used in the regimen (11-13); however, the irradiation has been reported to affect the neuroendocrine system in children and their subsequent growth and development (14), in addition to increasing the risk of malignancies.

IST is used to treat patients that lack a histocompatible donor or elderly patients, and ATG combined with cyclosporine remains the standard procedure for IST (6). Hematological responses to transfusion independence appear in $\sim 2 / 3$ of patients; however, disease relapse ultimately occurs in $30-40 \%$ of patients $(1,4)$. A 10 -year follow-up study revealed that the response rate of SAA is $71 \%$ and the actual event-free survival is $58 \%$ in 44 treatment-naïve patients with SAA that received high-dose cyclophosphamide (15). The success of IST application is significantly limited by poor response, high relapse and clonal evolution. In a previous study, the present authors treated patients with enhanced immunosuppressive treatment (100 mg/kg CTX) and UCB transfusion as an adjuvant therapy (5). Patients rapidly achieved reconstitution of hematopoiesis, while the efficacy rate was $88 \%$ and the 3-year overall survival rate was $92 \%$ (5). However, there were no signs of implant, so the therapy was named intensive IST combined with UCB transfusion, rather than UBCT (16). Furthermore, in a previous study, CTX (200 mg/kg) and rabbit ATG $(7.5 \mathrm{mg} / \mathrm{kg} /$ day for 4 days) were used as a conditioning regimen in a pediatric patient with SAA that was receiving UBCT (17). The patient's 
neutrophil count reached $0.5 \times 10^{9} / 1$ on day 37 , while red blood cell and platelet transfusion independence were reached on days 50 and 52, respectively. The patient demonstrated stable mixed chimerism at 18 months and full donor chimerism at 20 months after UCBT (17). The doses of CTX and ATG employed in the present cases were half of the previous dosages (18), and the adult patient received a reduced number of total nucleated cells and $\mathrm{CD}_{3} 4^{+}$cells compared with a previous study (18). However, the neutrophils and platelet engraftment recovered faster compared with those in the previous study. Furthermore, the appearance of full donor chimerism occurred earlier and without relapse during the 2-year follow-up period. Though the treatment may induce a higher risk of rejection due to the unrelated and mismatched UBC, the engraftment in two patients in the present study was successful.

In conclusion, the modified protocol used in the present study, which involved no irradiation, resulted in mixed chimerism and rapidly achieved complete reconstitution of hemopoiesis. The present report suggested the feasibility and efficacy of enhanced IST combined with UCB for the treatment of patients with SAA. Thus, the improved therapy may be a viable therapeutic option for patients that lack a suitable HLA-matched donor and may enhance the response rate of IST.

\section{Acknowledgements}

The authors would like to thank Dr Qing-Qing Yu and Dr Xiao-Chen Song of the General Hospital of Jinan Military (Jinan, China) for their assistance.

\section{References}

1. Rosenfeld S,Follmann D, Nunez O and Young NS: Antithymocyte globulin and cyclosporine for severe aplastic anemia: Association between hematologic response and long-term outcome. JAMA 289: 1130-1135, 2003

2. Scheinberg P and Young NS: How I treat acquired aplastic anemia. Blood 120: 1185-1196, 2012.

3. Peffault de Latour R, Purtill D, Ruggeri A, Sanz G, Michel G, Gandemer V, Maury S, Kurtzberg J, Bonfim C, Aljurf $\mathrm{M}$, et al: Influence of nucleated cell dose on overall survival of unrelated cord blood transplantation for patients with severe acquired aplastic anemia: A study by eurocord and the aplastic anemia working party of the European group for blood and marrow transplantation. Biol Blood Marrow Transplant 17: 78-85, 2011.
4. Frickhofen N, Heimpel H, Kaltwasser JP and Schrezenmeier H; German Aplastic Anemia Study Group: Antithymocyte globulin with or without cyclosporin A: 11-year follow-up of a randomized trial comparing treatments of aplastic anemia. Blood 101: 1236-1242, 2003.

5. Zhou F, Ge L, Yu Z, Fang Y and Kong F: Clinical observations on intensive immunosuppressive therapy combined with umbilical cord blood support for the treatment of severe aplastic anemia. J Hematol Oncol 4: 27, 2011.

6. Scheinberg P: Aplastic anemia. Therapeutic updates in immunosuppression and transplantation. Hematology Am Soc Hematol Educ Program 2012: 292-300, 2012.

7. Marsh J: Making therapeutic decisions in adults with aplastic anemia. Hematology Am Soc Hematol Educ Program: 78-85, 2006.

8. Broxmeyer HE, Douglas GW, Hangoc G, Cooper S, Bard J, English D, Arny M, Thomas L and Boyse EA: Human umbilical cord blood as a potential source of transplantable hematopoietic stem/progenitor cells. Proc Natl Acad Sci USA 86: 3828-3832, 1989.

9. Gluckman E, Rocha V,Boyer-Chammard A,Locatelli F, Arcese W Pasquini R, Ortega J, Souillet G, Ferreira E, Laporte JP, et al: Outcome of cord-blood transplantation from related and unrelated donors. Eurocord transplant group and the european blood and marrow transplantation group. N Engl J Med 337: 373-381, 1997.

10. MacMillan ML, Walters MC and Gluckman E: Transplant outcomes in bone marrow failure syndromes and hemoglobinopathies. Semin Hematol 47: 37-45, 2010.

11. Lee JW, Kang HJ, Kim EK, Shin HY and Ahn HS: Successful salvage unrelated umbilical cord blood transplantation with two units after engraftment failure with single unit in severe aplastic anemia. J Korean Med Sci 24: 744-746, 2009.

12. Yoshimi A, Kojima S, Taniguchi S, Hara J, Matsui T, Takahashi Y, Azuma H, Kato K, Nagamura-Inoue T, Kai S and Kato S; Japan Cord Blood Bank Network: Unrelated cord blood transplantation for severe aplastic anemia. Biol Blood Marrow Transplant 14: 1057-1063, 2008

13. Yamamoto H, Kato D, Uchida N, Ishiwata K, Araoka H, Takagi S, Nakano N, Tsuji M, Asano-Mori Y, Matsuno N, et al: Successful sustained engraftment after reduced-intensity umbilical cord blood transplantation for adult patients with severe aplastic anemia. Blood 117: 3240-3242, 2011.

14. Sanders JE: Growth and development after hematopoietic cell transplant in children. Bone Marrow Transplant 41: 223-227, 2008.

15. Brodsky RA, Chen AR, Dorr D, Fuchs EJ, Huff CA, Luznik L, Smith BD, Matsui WH, Goodman SN, Ambinder RF and Jones RJ: High-dose cyclophosphamide for severe aplastic anemia: Long-term follow-up. Blood 115: 2136-2141, 2010.

16. Xie LN, Fang Y, Yu Z, Song NX, Kong FS, Liu XM and Zhou F: Increased immunosuppressive treatment combined with unrelated umbilical cord blood infusion in children with severe aplastic anemia. Cell Immunol 289: 150-154, 2014.

17. Boiron JM, Cotterêt S, Cony-Makhoul P, Merel P, Micheau M, Perel Y, Belloc F, Bernard P and Reiffers J: Stable mixed chimerism without relapse after related allogeneic umbilical cord blood transplantation in a child with severe aplastic anemia. Bone Marrow Transplant 22: 819-821, 1998.

18. Laughlin MJ, Barker J, Bambach B, Koc ON, Rizzieri DA, Wagner JE, Gerson SL, Lazarus HM, Cairo M, Stevens CE, et al: Hematopoietic engraftment and survival in adult recipients of umbilical-cord blood from unrelated donors. N Engl J Med 344: 1815-1822, 2001. 